\title{
Association learning and pitch perception
}

\author{
ROBERT S. BUNDY \\ Boulder, Colorado \\ JOHN COLOMBO \\ University of Kansas, Lawrence, Kansas \\ and \\ PATRICIA WARNICK-YARMEL \\ University of Illinois, Chicago, Illinois
}

\begin{abstract}
In two experiments, subjects matched the pitch of a set of low-component harmonic complexes to that of a higher component complex, were then exposed for $1 \mathrm{~h}$ to complex tones in which low harmonics were tuned $3 \%$ sharp or $3 \%$ flat (relative to higher ones), and then repeated pitch matching. Comparison of pre- and postexposure pitch-matching performance indicated that exposure to sharp-tuned harmonics caused subjects to match higher on the posttest and exposure to flattuned harmonics caused them to match lower. These data support the notion that normal pitch perception may develop and be maintained as a function of experience with normally occurring sounds.
\end{abstract}

Adult subjects can extract an invariant pitch from a wide variety of acoustic waveforms. One example of this is the missing fundamental (MF) phenomenon (Wightman \& Green, 1974). Naturally occurring sounds usually possess some energy at a fundamental frequency $\left(\mathrm{F}_{0}\right.$, e.g., $200 \mathrm{~Hz})$ and higher harmonics at integer multiples of $F_{0}$ (e.g., $400,600,800,1000 \mathrm{~Hz}$ ). If presented with a tone comprised of, for example, harmonics 2 through 5 (i.e., $400-600-800-1000 \mathrm{~Hz}$ ), subjects will accurately match the missing $\mathrm{F}_{\mathrm{o}}$ (i.e., the first harmonic, $200 \mathrm{~Hz}$ ) to that tone.

Early theories of this phenomenon posited that the missing $\mathrm{F}_{\mathrm{o}}$ was extracted from the physical properties of the harmonic complex. For example, the waveform envelope produced by summing sine wave components of 400-600$800-1000 \mathrm{~Hz}$ actually repeats at $200 \mathrm{~Hz}$, thus providing a cue from which $F_{0}$ might be extracted (deBoer, 1956). However, the accuracy of MF performance is not impaired when this periodicity is destroyed by shifting the phase of the summed harmonic components (Patterson, 1973). Subsequent theories proposed that $F_{0}$ was determined by the mathematical relationship among the components through, for example, a statistical "best fit" analysis based on the harmonics of the complex stimulus (Goldstein, 1973).

However, the MF task has been likened to visual pattern recognition tasks in which the absence of a particular feature of a complex form does not impede recognition of the form as a whole. Similarly, the absence of one particular feature (i.e., $F_{0}$ ) of a complex auditory stimulus may not hinder the recognition of that stimulus' pitch

Results of these experiments were presented at the meeting of the Acoustical Society of America in Ottowa, Ontario, March 1981. Reprint requests may be sent to John Colombo, Department of Human Development, University of Kansas, Lawrence, KS 66045.
(Wightman, 1973a, 1973b). One possible implication of this is that experience with naturally occurring sounds may be necessary for the development or maintenance of the association between the various acoustic components that comprise complex sounds. This position is supported by some research with both human infants (Bundy, Colombo, \& Singer, 1982) and adults (Hall \& Peters, 1982; Hall $\&$ Soderquist, 1982). One prediction of the experiencedbased theories is that prolonged exposure to mistuned harmonic complexes should affect subjects' performance in MF tasks. In the following experiments, subjects were exposed for $1 \mathrm{~h}$ to complex tones in which a subset of harmonics was mistuned by $3 \%$ with respect to the other components of the waveform. The subjects' MF task performance was assessed within a pre/post design in order to evaluate the effects of this exposure on pitch perception.

\section{EXPERIMENT 1}

\section{Method}

Subjects. Eighty-three students were screened for accuracy in matching the pitch of two harmonic complexes (see below). Fourteen of these subjects attained a $S D$ error of $1.0 \%$ on this task, and were invited back for an experimental session.

Stimuli. Stimuli were generated with a Gimix 6809 microcomputer through an 8-bit D/A converter (13.5-kHz sample rate). For presentation, signals were low-passed through a $5-\mathrm{kHz}$ filter $(48 \mathrm{~dB} /$ octave rolloff), amplified, and fed to an ADS 100 speaker $30 \mathrm{~cm}$ in front of the subject at an intensity of $75 \mathrm{~dB}\left(\mathrm{re}=0.0002 \mathrm{dyne} / \mathrm{cm}^{2}\right)$. Rise/fall times were $10 \mathrm{msec}$.

Pitch match stimuli: In screening, during pre- and postexposure tests, the subjects were asked to match the pitch of the 8 halftones from middle $\mathrm{C}$ through the $\mathrm{G}$ above middle $\mathrm{C}$ by adjusting the lower of two alternating tones (500-msec duration each). The lower, adjustable tone was composed of harmonics 1 to 3 ; the higher tone was composed of harmonics 4 to 7 .

Exposure stimuli: During the exposure period, harmonic components for the same $\mathbf{8}$ halftones used in pitch matching were constructed from 
harmonics 1-7 summed at equal intensity in random phase. For half of the subjects (the "up," or U, group), the frequencies of harmonics 1-3 were adjusted sharp by $3 \%$, relative to the partials $4-7$, and for the other half (the "down," or D, group), harmonics 1-3 were adjusted flat by $3 \%$ relative to harmonics 4-7. That is, for a normal complex tone composed of the components $200-400-600-800-1000-1200-1400 \mathrm{~Hz}$, the U group heard the components 216-412-618-800-1000-1200$1400 \mathrm{~Hz}$, and the $\mathrm{D}$ group heard the components 194-388-582-800-1000-1200-1400 Hz.

Procedure. The experimental design consisted of a pitch-matching task both before and after an exposure period to mistuned stimuli.

Pitch-matches: In screening, during pre- and postexposure tests, the subjects, using a knob, adjusted the pitch of the lower of two alternating harmonic complexes until satisfied with the accuracy of the match (no time constraint was imposed). The subjects pressed a button when attaining such a match, which signaled the computer to store the value of the match and to present another pair of tones. Each of the 8 halftones were presented in three randomized blocks for a total of 24 trials.

Exposure period: For $1 \mathrm{~h}$, the subjects participated in a task modeled after a popular note-sequence matching game. The computer generated a random sequence of tones (selected from the range from middle $\mathrm{C}$ to next $G$ ), which the subjects were asked to match on a regular-size Moog keyboard. The subjects could vary the difficulty of the task by selecting the number of notes in the sequence (1 to 9 ) and the similarity of the timbre of the keyboard tones to that of the presented sequence (by varying the relative intensities of harmonics 1-3 and 4-7). Such variation exposed the subjects to various pitch strengths from which featural invariants could be abstracted. The subjects were instructed to work at their own pace and at the most difficult levels they could, and typically made several matches per minute. To encourage active participation and attention to the task, the subjects received feedback on their matches, and the experimenters implied that their progress was being monitored. A posttest pitch-matching task immediately followed the exposure period.

\section{Results and Discussion}

The subjects' individual pre- and postexposure pitchmatching performance is presented in Figure 1. Estimates were fairly consistent within individuals but varied widely between subjects (see Terhardt, 1974). A group $\times$ pre/post $\times$ frequency $\times$ trial ANOVA on the subjects' pitch matches yielded a significant $(p<.001)$ main effect for frequency (i.e., the subjects matched different pitches to different harmonic complexes), and a significant group $\times$ pre/post interaction $[F(1,12)=9.8$, $p<.01]$. The interaction is attributable to the alteration of the subjects' pitch matches in the direction that lower harmonics had been modified during the exposure period. Individual changes from pre- to postexposure tests were statistically significant (in the predicted direction) in 9 of the 14 cases.

The results clearly support the experiential hypothesis. However, as Figure 1 indicates, all subjects' estimates were flat. This might have been due to the presence of higher frequency harmonics (i.e., 2-3) in the lower pitchmatch tone, as predicted by certain pitch-extraction models (Terhardt, 1971; Terhardt, Stoll, \& Seewann, 1982). Experiment 2 was conducted to test this possibility and to replicate the exposure effect with another set of stimuli.

\section{EXPERIMENT 2}

\section{Method}

Ten subjects were selected from a screened sample of 52, on the basis of the same criterion as in Experiment 1. Stimuli were synthesized and presented exactly as in Experiment 1, with the following exceptions. First, in screening, during preexposure and postexposure pitch matches, the subjects matched $F_{0}$ s to tones comprised of partials 2-7. Second, during the exposure period, the subjects were presented with tones in which only $\mathrm{F}_{0}$ was tuned sharp or flat by $3 \%$. Difficulty levels

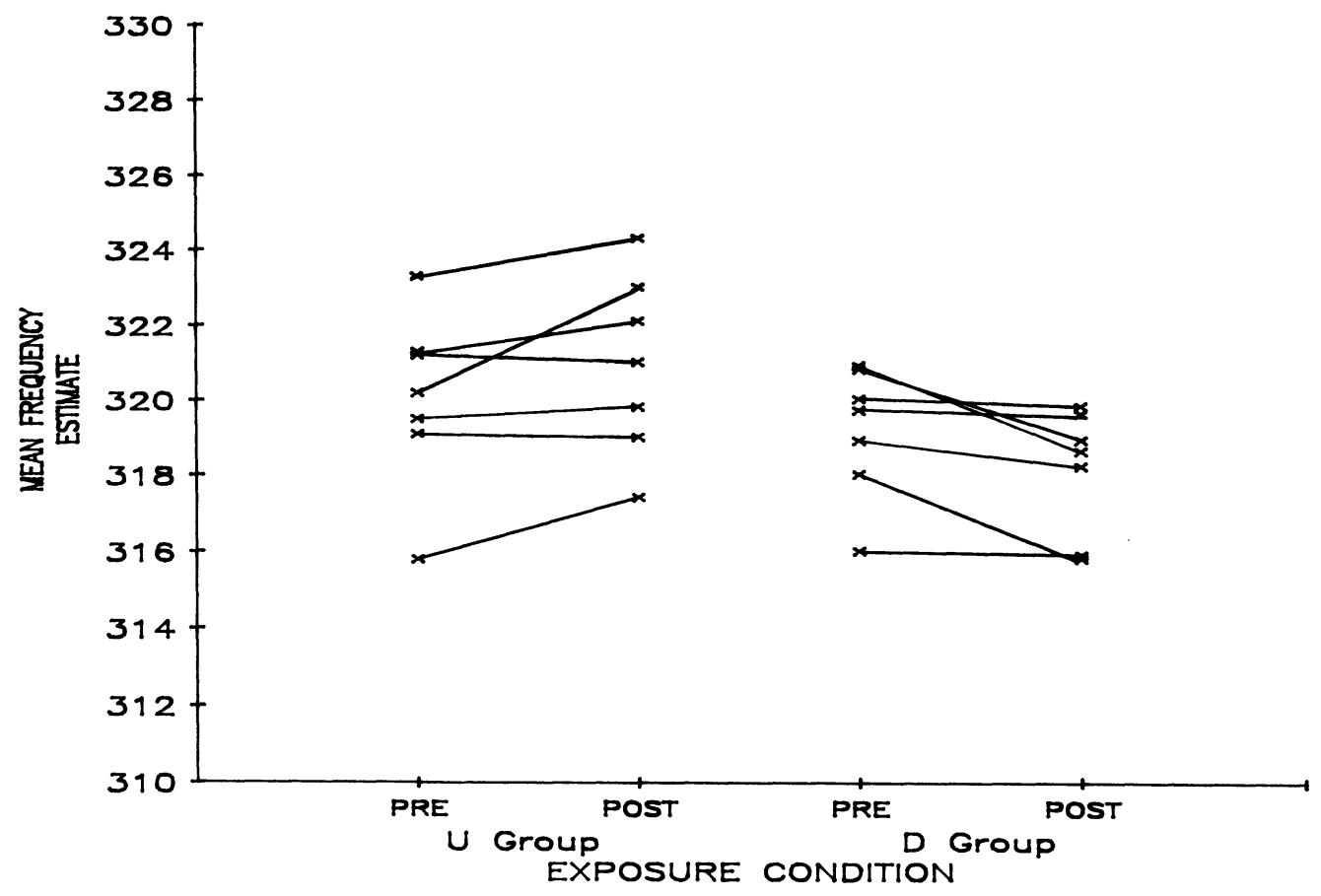

Figure 1. Results of individual subjects tested in Experiment 1. Each point represents the mean of one subject's performance collapsed across three trials at eight different frequencies. (Subjects are matching to an actual mean frequency of $323 \mathrm{~Hz}$.) 


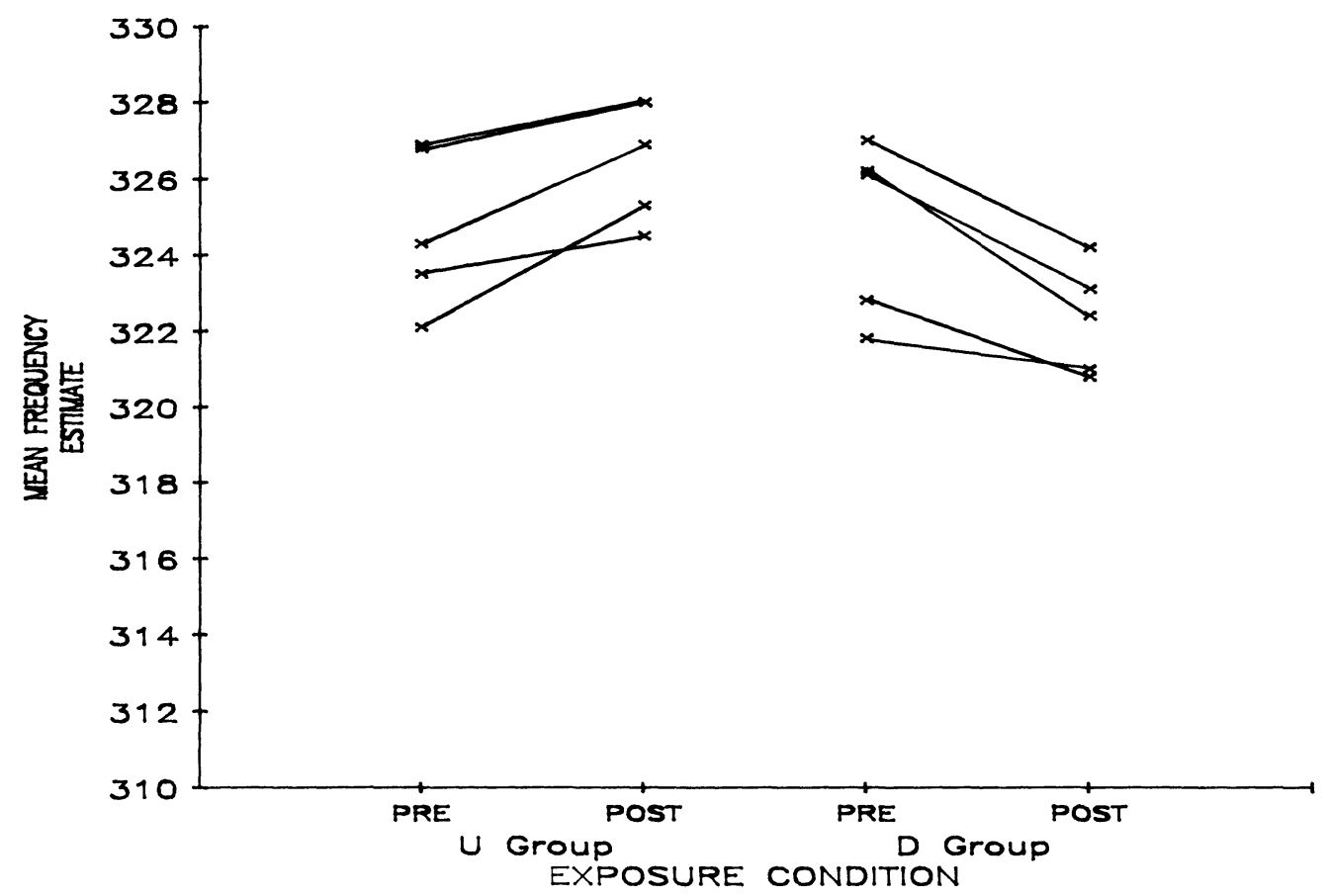

Figure 2. Results of individual subjects tested in Experiment 2. (Subjects are matching to an actual mean frequency of $323 \mathrm{~Hz}$.)

in matching note sequences was varied by manipulating the relative intensity of $F_{0}$ versus partials 2-7. Procedures for screening, pretests, exposure, and posttests for Experiment 2 were exactly as in Experiment 1 .

\section{Results and Discussion}

The subjects' individual pre- and postexposure pitchmatching performance is presented in Figure 2. As in Experiment 1 , a group $\times$ pre/post $\times$ frequency $\times$ trial ANOVA yielded a significant $(p<.001)$ main effect for frequency and a significant group $\times$ pre/post interaction $[F(1,8)=12.8, p<.01]$. The subjects again altered their pitch matches in the direction that $F_{0}$ had been modified during the exposure period. Individual changes were statistically significant in 8 of 10 cases. Additionally, as is evident from Figure 2, changes in pitch-match complexes eliminated the flatness of pitch estimates observed in Experiment 1.

\section{GENERAL DISCUSSION}

The data from these two experiments strongly suggest the manipulability of periodicity pitch. In tandem with research that demonstrates the absence of periodicity pitch in young infants (Bundy et al., 1982), these studies provide support for the notion that normal pitch perception may be established through, and dependent upon, some associative learning process (Hall \& Peters, 1982; Terhardt, 1974).

\section{REFERENCES}

Bundy, R., Colombo, J., \& Singer, J. (1982). Pitch perception in young infants. Developmental Psychology, 18, 10-14.

DEBOER, E. (1956). On the residue of hearing. Unpublished doctoral dissertation, University of Amsterdam.

Goldstein, J. (1973). An optimum processor theory for the central formation of the pitch of complex tones. Journal of the Acoustical Society of America, 54, 1496-1514.

Hall, J., \& Peters, R. (1982). Change in the pitch of a complex tone following its association with a second complex tone. Journal of the Acoustical Society of America, 71, 142-147.

Hall, J., \& SoDERQUIST, D. (1982). Transient complex and pure tone pitch changes by adaptation. Journal of the Acoustical Society of America, 71, 665-670.

Patterson, R. (1973). The effects of relative phase and the number of components on residue pitch. Journal of the Acoustical Society of America, 53, 1565-1572.

TerhardT, E. (1971). Die tonhohe harmonischer Klange und das Oktavintervall. Acoustica, 24, 126.

TeRHARDT, E. (1974). Pitch, consonance, and harmony. Journal of the Acoustical Society of America, 54, 1061-1069.

Terhardt, E., Stoll, G., \& Seewann, M. (1982). Pitch of complex signals according to virtual-pitch theory: Tests, examples, and predictions. Journal of the Acoustical Society of America, 71, 671-678.

Wightman, F. (1973a). The pattern transformation model of pitch. Journal of the Acoustical Society of America, 54, 407-416.

Wightman, F. (1973b). Pitch and stimulus fine structure. Journal of the Acoustical Society of America, 54, 417-423.

Wightman, F., \& Green, D. (1974). The perception of pitch. American Scientist, 54, 407-416. 